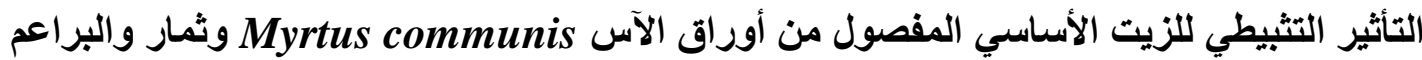

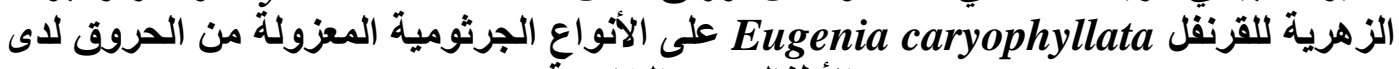

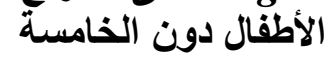

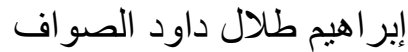

فرع العلوم التمريضية الأساسية / كلية التمريض / الهرافة الموصل / العراق

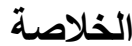

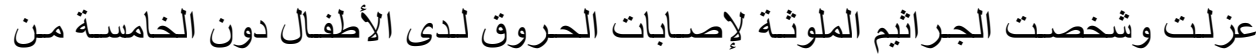

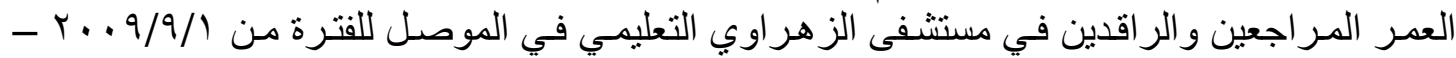

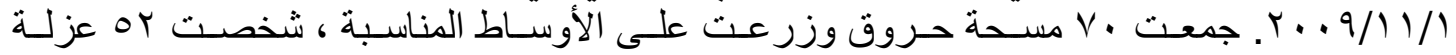

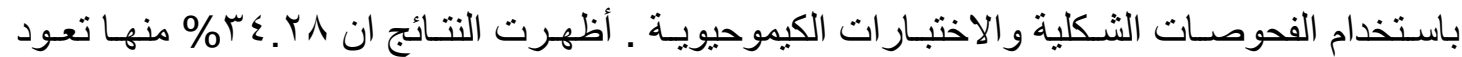

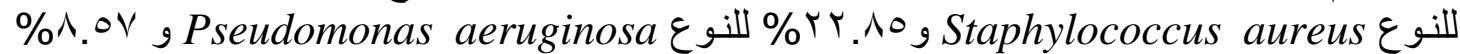

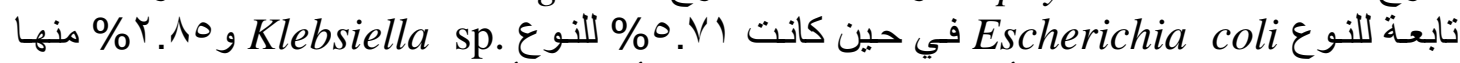

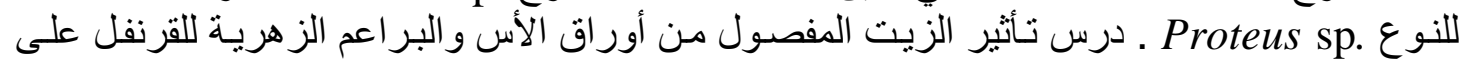

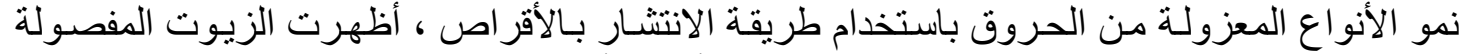

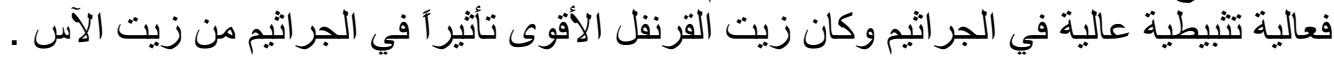

\section{المقدمة}

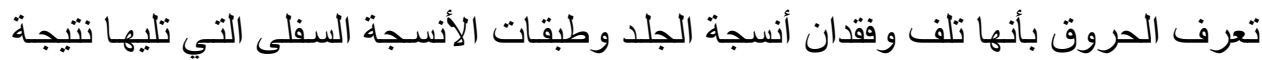

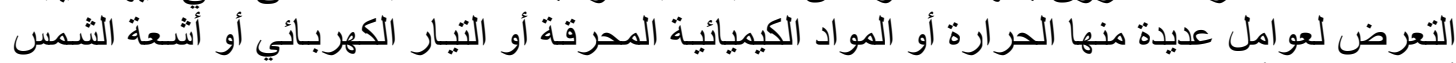

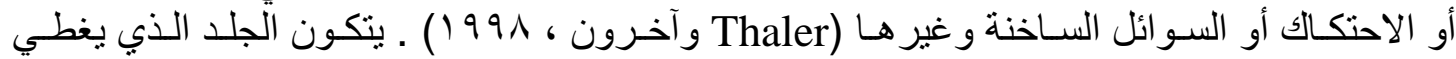

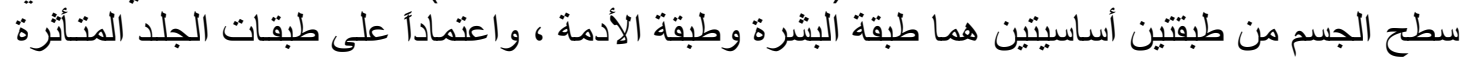

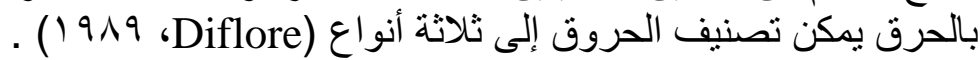

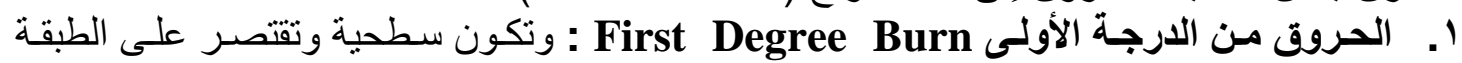

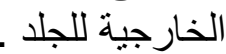

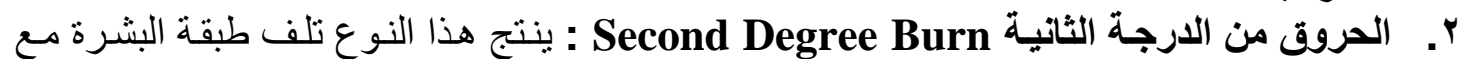

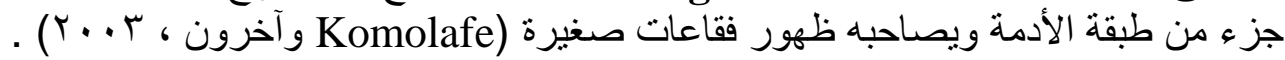

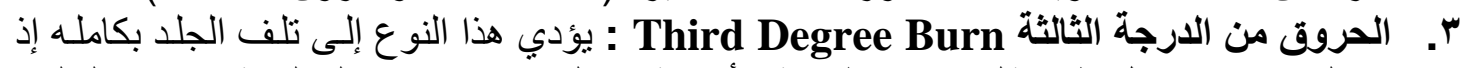

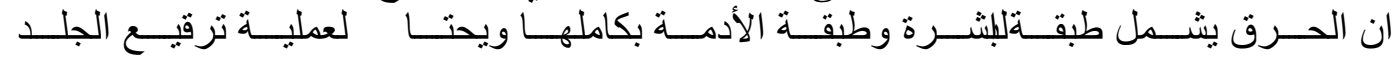
(Paint) بعد حدوث الحرق وإزالة الحاجز الميكانيكي (الجلا) تصبح منطقة الإصابة حساسة جداً لغزو الأحياء

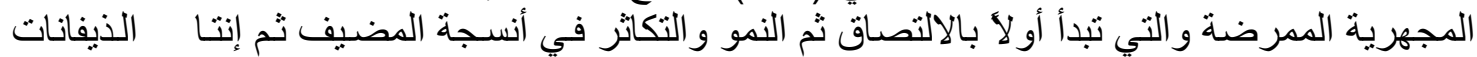

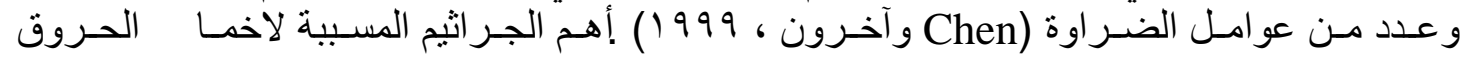
هـ Streptococcus sp. و و و

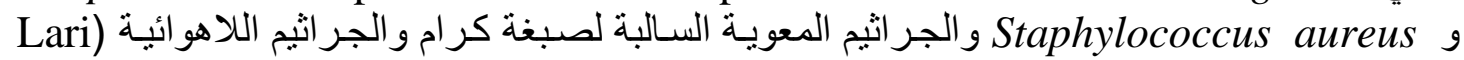

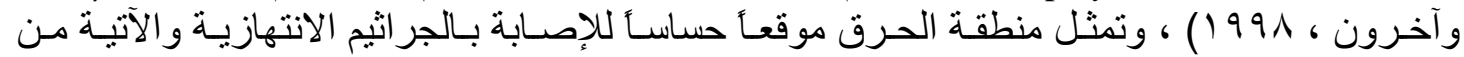

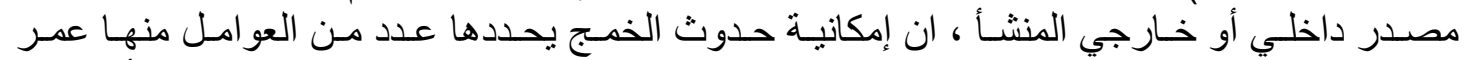

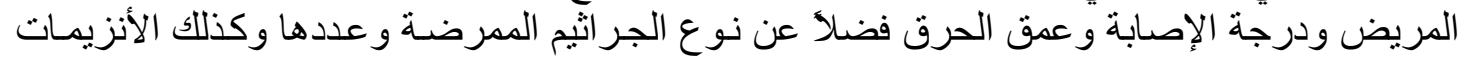

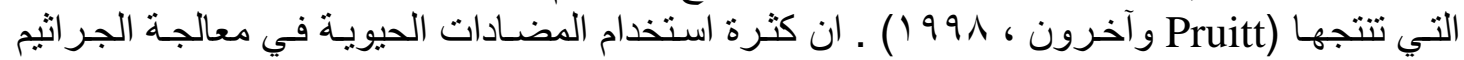

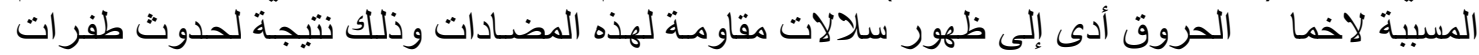

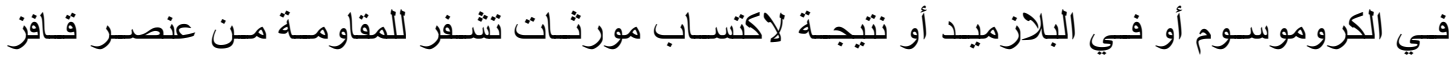
(Transposon)

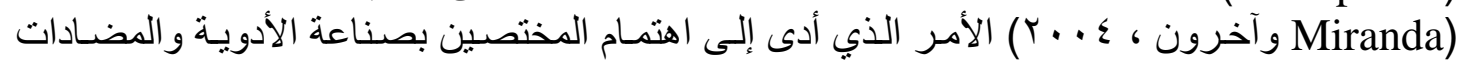

$$
\text { تاريخ تسلم البحث }
$$




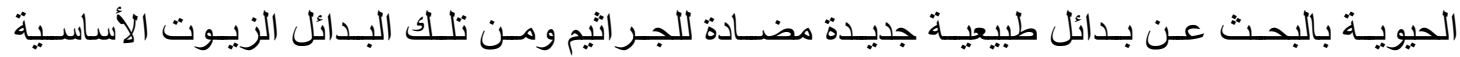

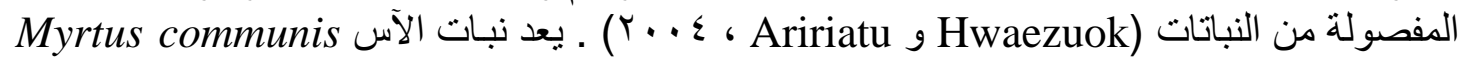

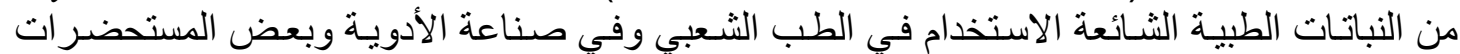

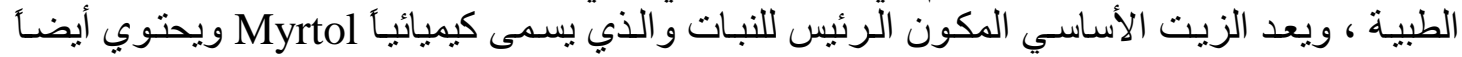

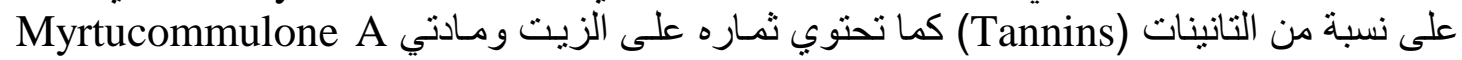

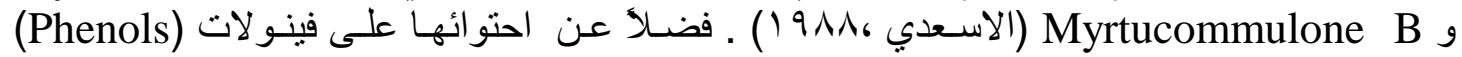

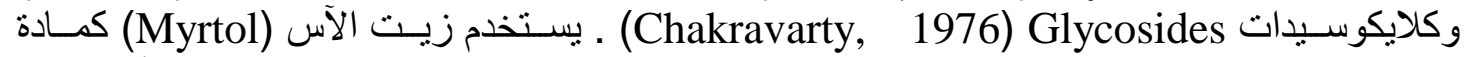

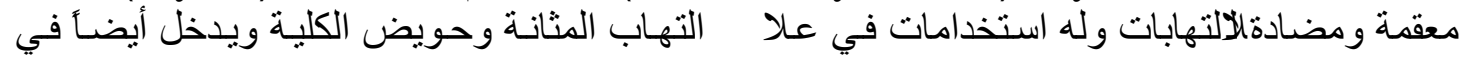

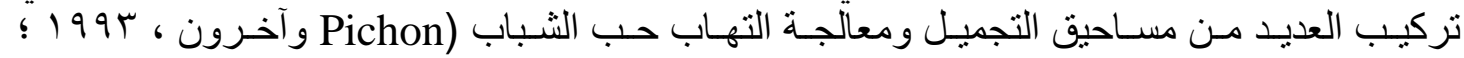
Evans و آخرون ، $99 \mathrm{~V}$

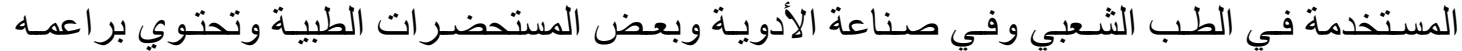

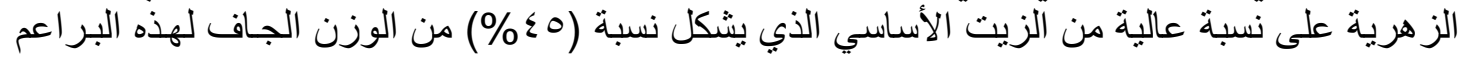

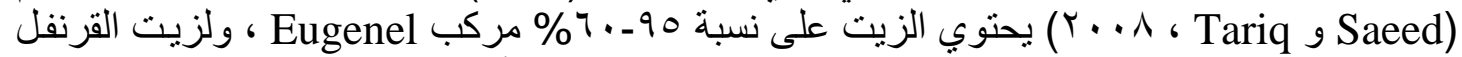

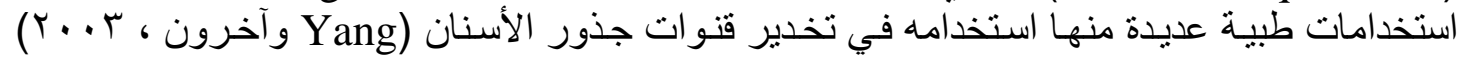

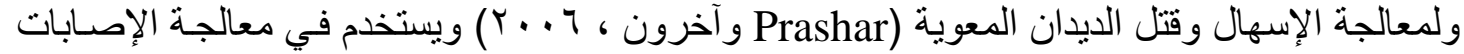
ة

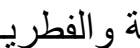
الجرثومي

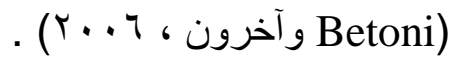

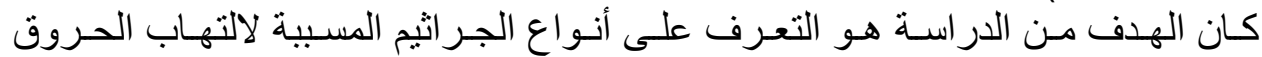

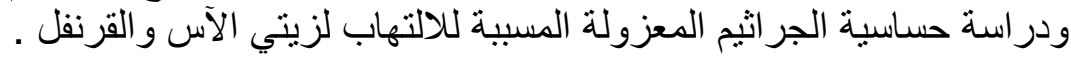

\section{مواد البحث وطرائقه}

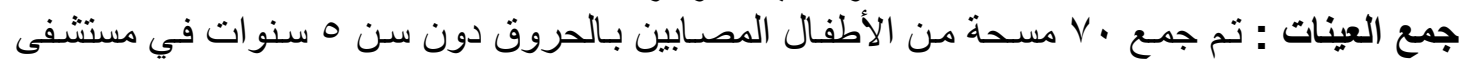

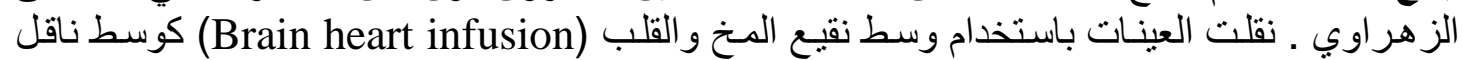

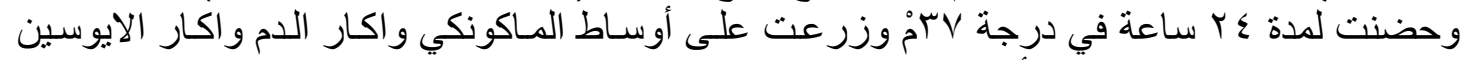

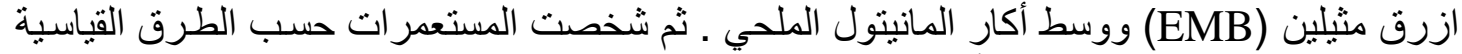

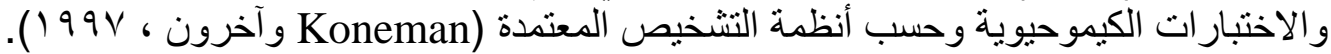

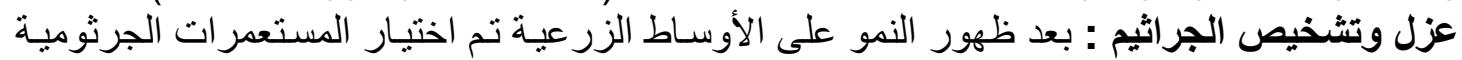

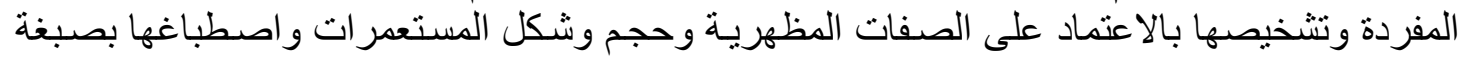

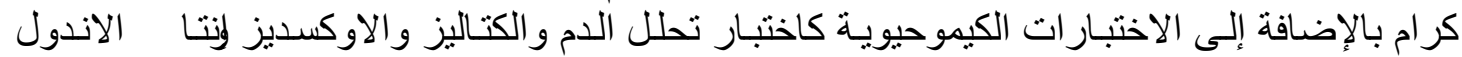

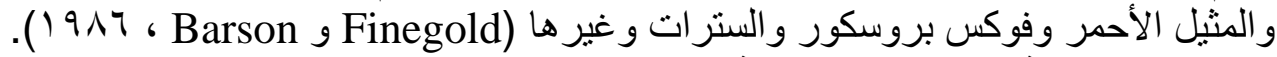

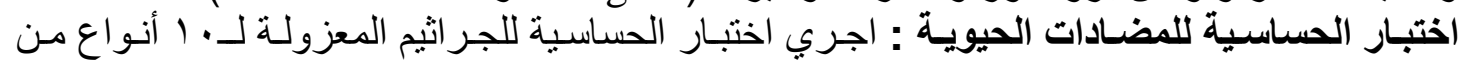

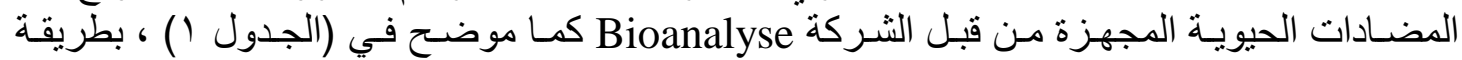

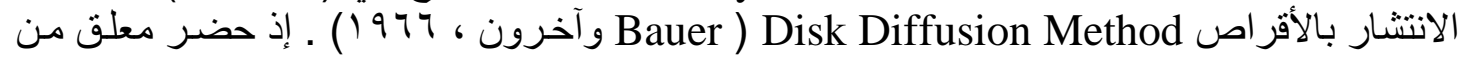

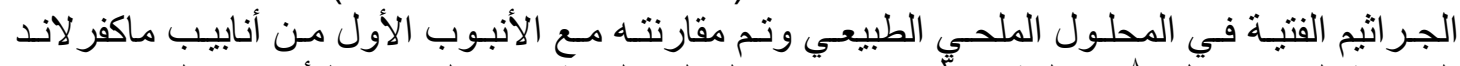

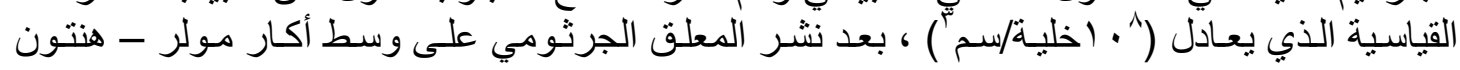
(Muller Hinton agar)

الجدول ( () : أنواع وتر اكيز المضادات الحيوية المستخدمة بالدراسة

\begin{tabular}{|c|c|c|c|c|c|}
\hline الرمز ل & 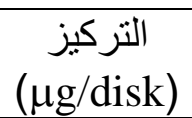 & اسم المضناد & الرمز & $\begin{array}{c}\text { التركيز } \\
\text { ( } \mu \text { g/disk) }\end{array}$ & اسم المضاد \\
\hline TE & $\mu$ & Tetracyclin & $\mathrm{AK}$ & $\mu$ & Amikacin \\
\hline RA & 0 & Rifampicin & AP & 1. & Ampicillin \\
\hline $\mathrm{NA}$ & $r$. & Naldixic acid & $\mathrm{CE}$ & $r$. & Cephalexin \\
\hline $\mathrm{TB}$ & 1. & Tobramycin & $\mathrm{CP}$ & 0 & Ciprofloxacin \\
\hline $\mathrm{KN}$ & $r$. & Kanamycin & GN & 1. & Gentamicin \\
\hline
\end{tabular}




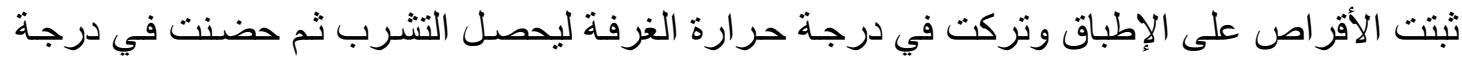

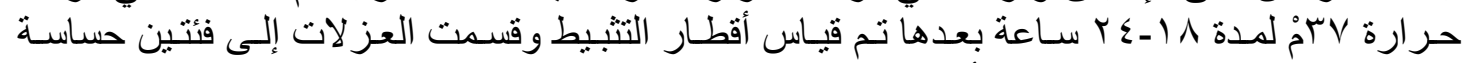

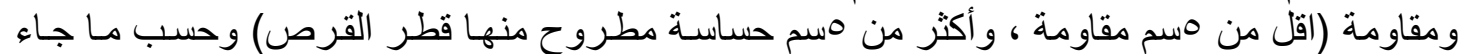

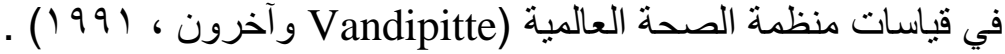

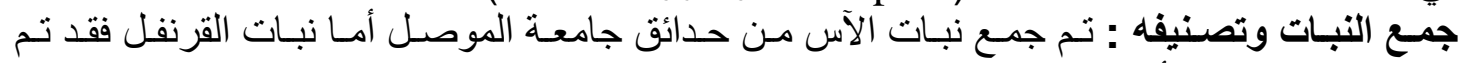

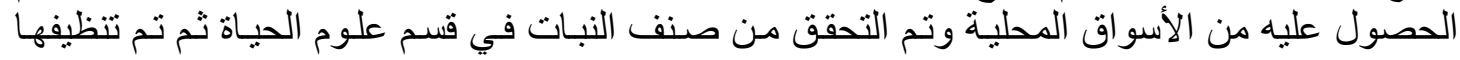

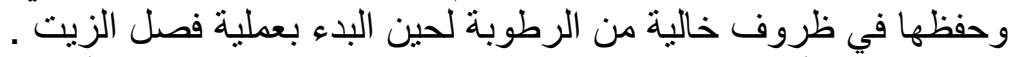

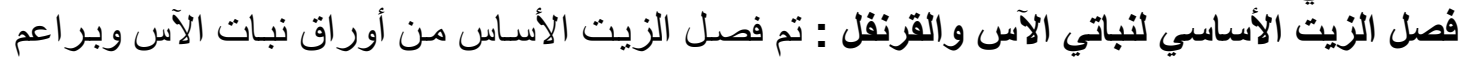

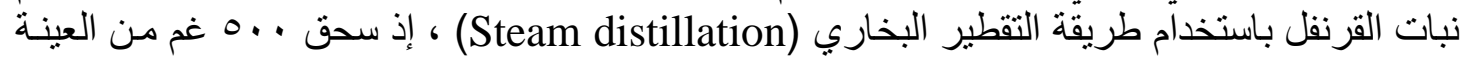

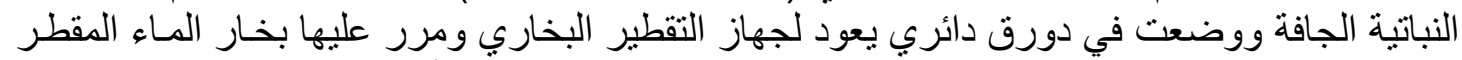

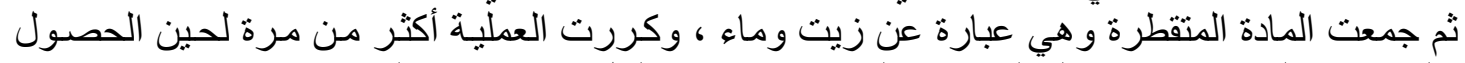

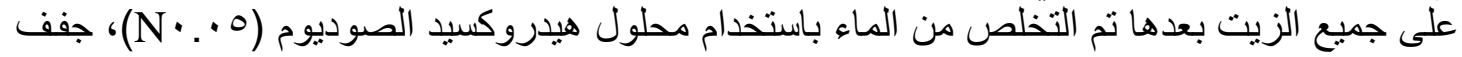

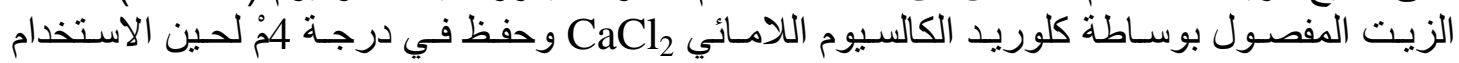

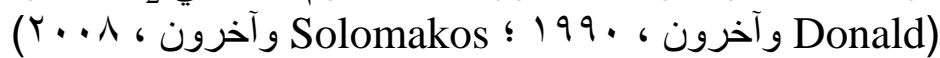

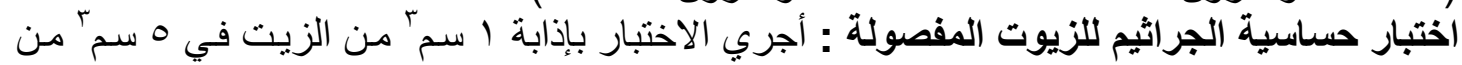

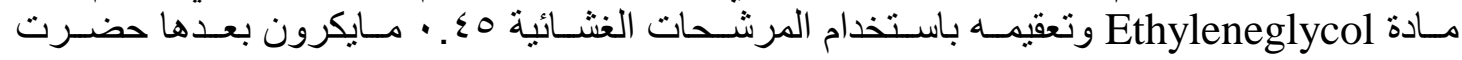

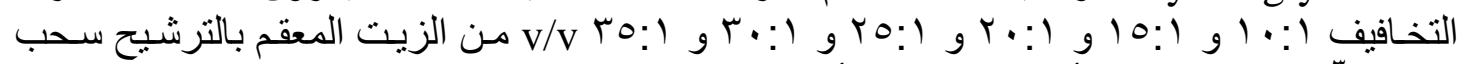

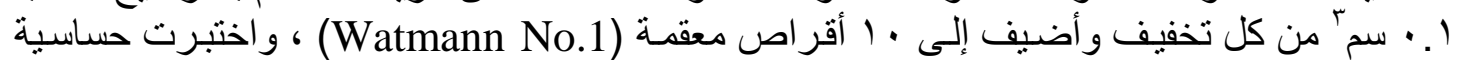

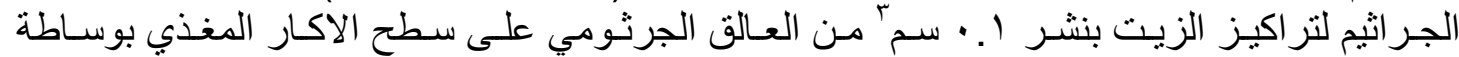

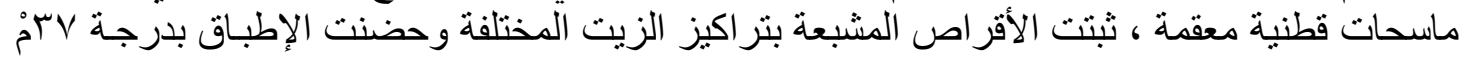

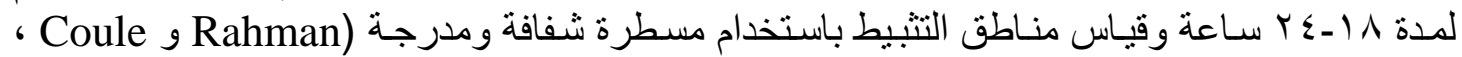
. ( r. r

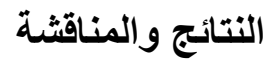

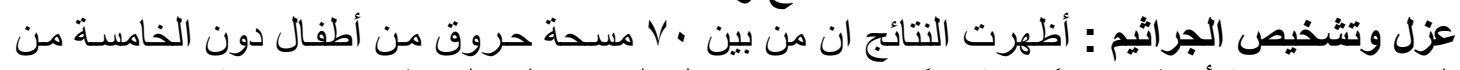

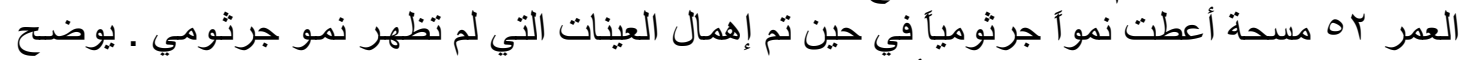

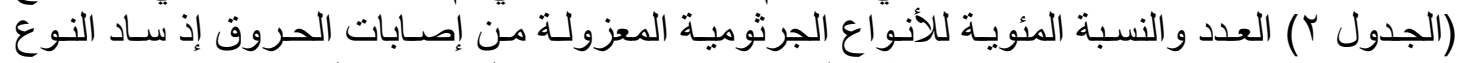
Staph. aureus

الجدول (Y): العدد و النسبة المئوية للجر اثيم المعزولة من إصابات الحروق للأطفال دون سن ه سنوات

\begin{tabular}{|c|c|c|}
\hline النسبة المئوية & 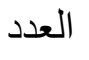 & 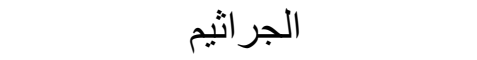 \\
\hline$\sum 7.0$ & rs & Staphylococcus aureus \\
\hline$r \cdot . V V$ & 17 & Pseudomonas aeruginosa \\
\hline $11.0 \varepsilon$ & 7 & Escherichia coli \\
\hline$V .79$ & $\varepsilon$ & Klebsiella spp. \\
\hline r.Ao & $r$ & Proteus spp. \\
\hline
\end{tabular}

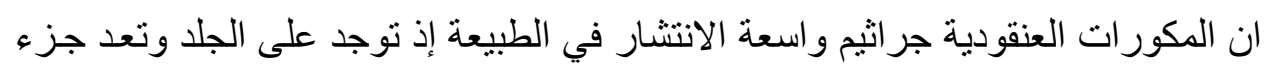

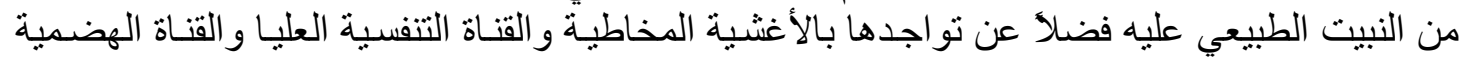

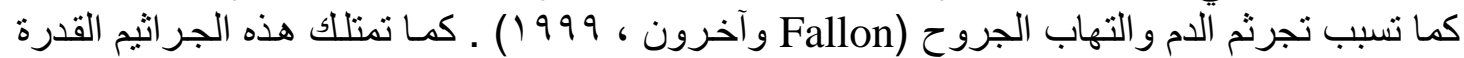

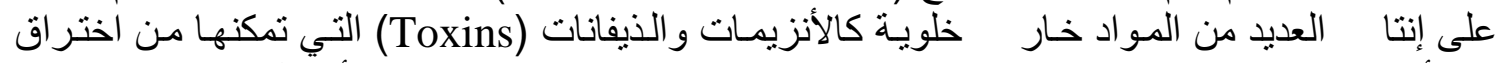

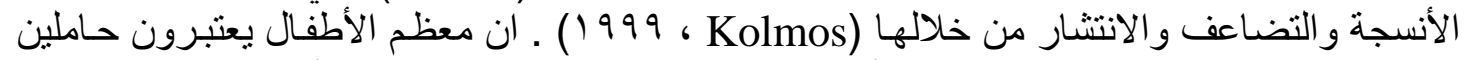

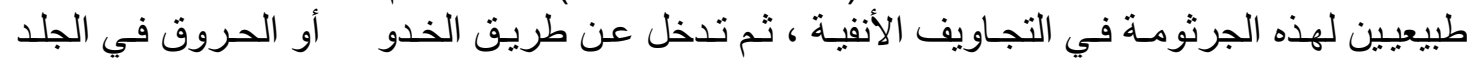

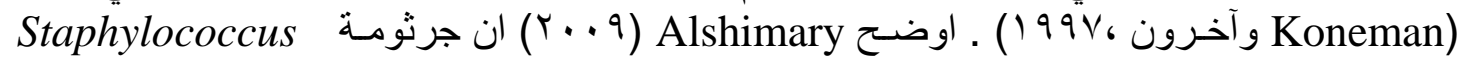
هoureus 


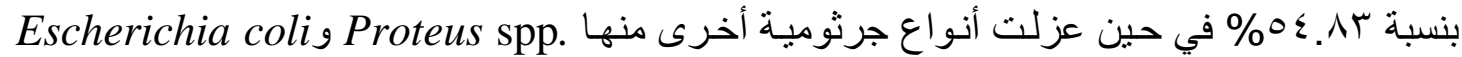

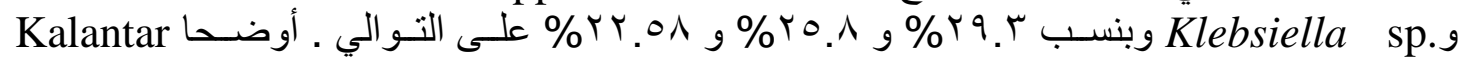

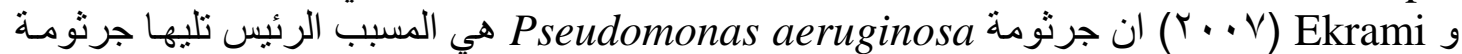
Staphylococcus aureus

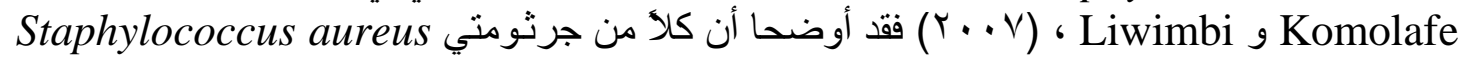

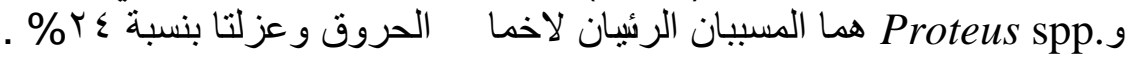

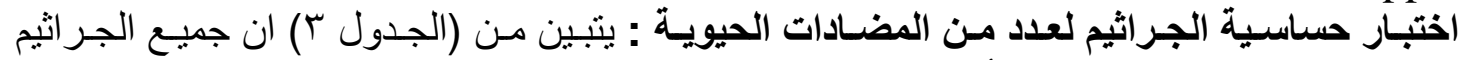

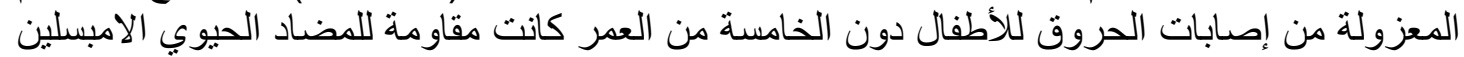

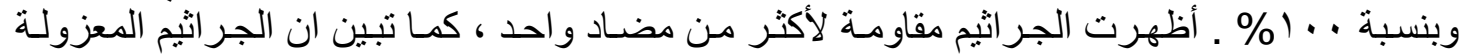

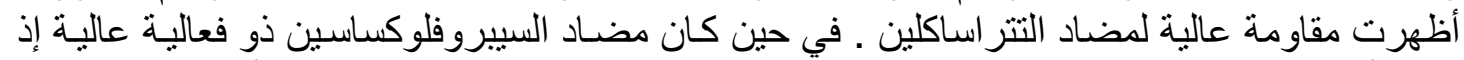

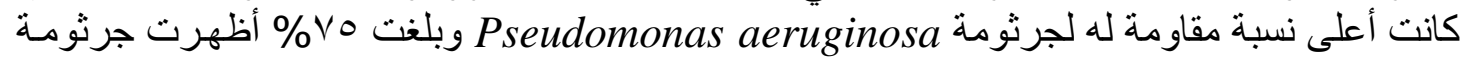

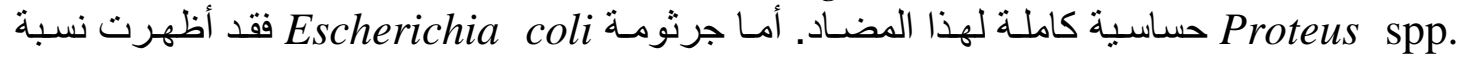

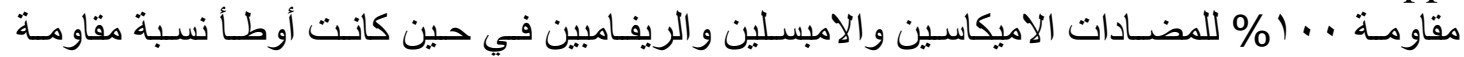

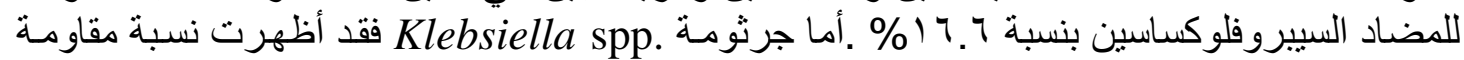

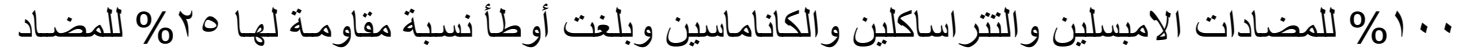

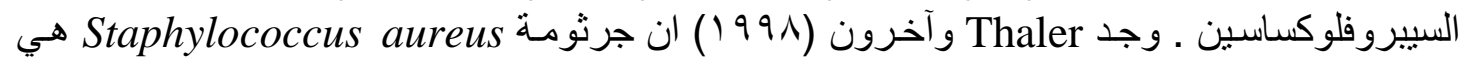

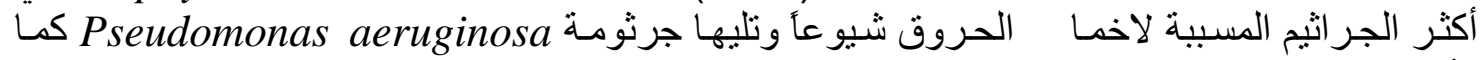

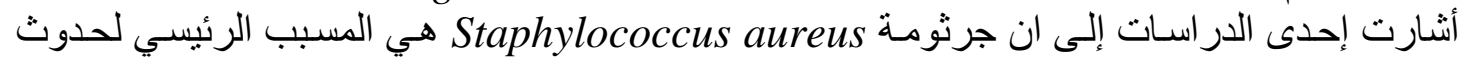

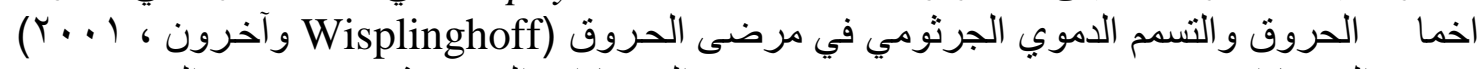

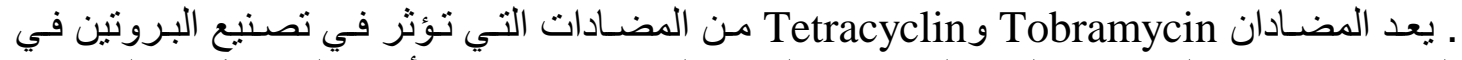

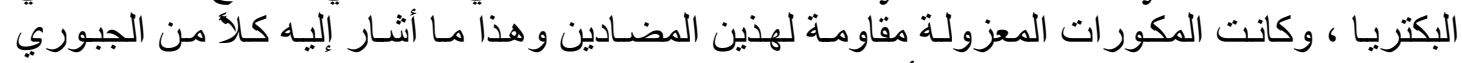

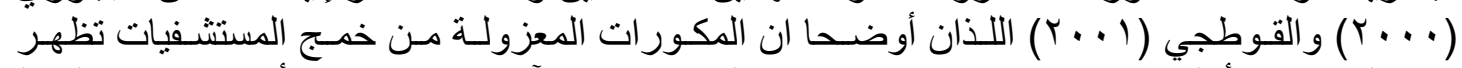

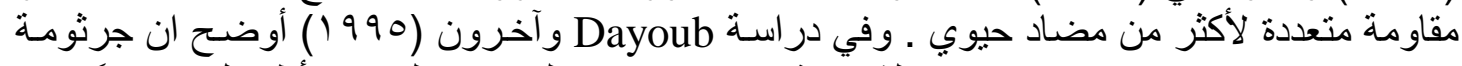
Staphylococcus aureus

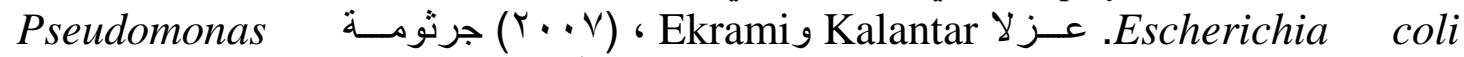
aeruginosa

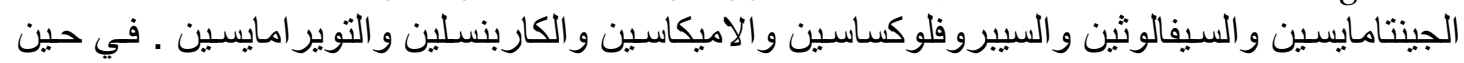

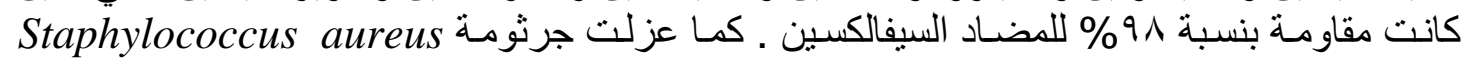

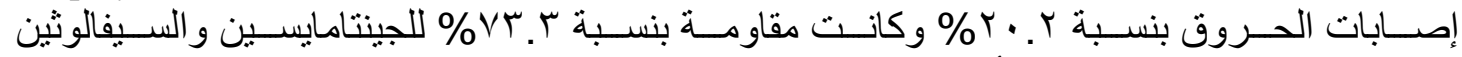

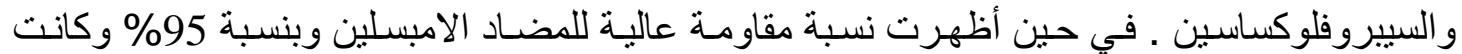

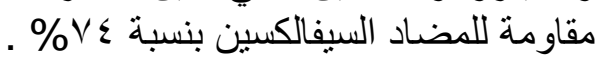

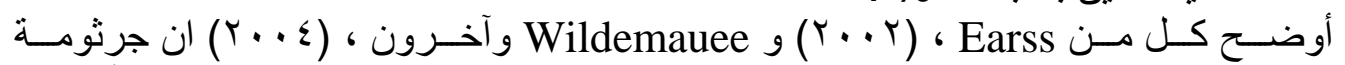
Staphylococcus aureus

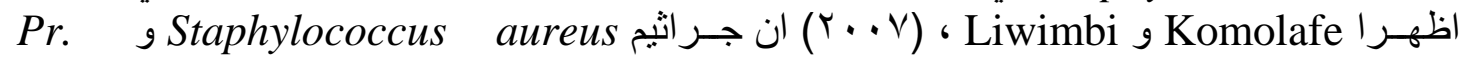
Streptococcus sp. و mirabilis

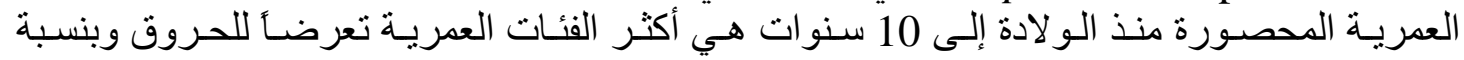

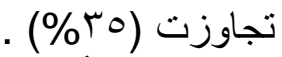

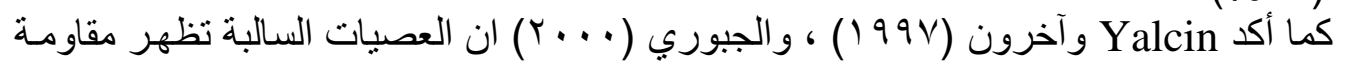

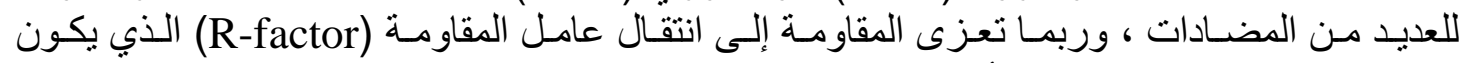

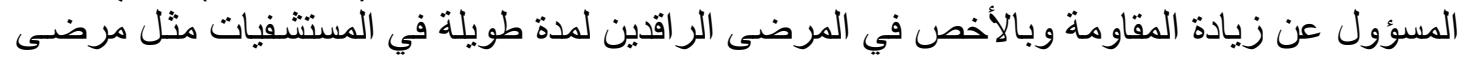

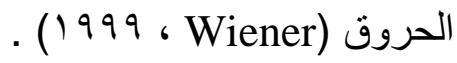


الجدول (r) : حساسية الجر اثثم الدعزولة من إصابات الحروق للمضادات الحيوية المستخدمة قبد الدراسة

\begin{tabular}{|c|c|c|c|c|c|c|c|c|c|c|c|c|c|c|c|c|c|c|c|c|c|}
\hline \multicolumn{2}{|c|}{ AK } & \multicolumn{2}{|c|}{ AP } & \multicolumn{2}{|c|}{$\mathrm{CE}$} & \multicolumn{2}{|c|}{$\mathrm{CP}$} & \multicolumn{2}{|c|}{ GN } & \multicolumn{2}{|c|}{ TE } & \multicolumn{2}{|c|}{ RA } & \multicolumn{2}{|c|}{ NA } & \multicolumn{2}{|c|}{$\mathrm{TB}$} & \multicolumn{2}{|c|}{$\mathrm{KN}$} & \multirow{2}{*}{ العدد ال العد } & \multirow{2}{*}{ الأنو اعز الجرثومية } \\
\hline $\mathrm{R}$ & $\mathrm{S}$ & $\mathrm{R}$ & $S$ & $\mathrm{R}$ & S & $\mathrm{R}$ & S & $\mathrm{R}$ & $\mathrm{S}$ & $\mathrm{R}$ & S & $\mathrm{R}$ & $\mathrm{S}$ & $\mathrm{R}$ & $\mathrm{S}$ & $\mathrm{R}$ & S & $\mathrm{R}$ & $\mathrm{S}$ & & \\
\hline $9 r$ & $\stackrel{i}{i}$ & $\begin{array}{l}r \leq \\
1 \cdots\end{array}$ & - & YTr & $\stackrel{i}{r}$ & 1T. & $\begin{array}{l}11 \\
\leq 0.1\end{array}$ & $\begin{array}{l}r^{\prime} \\
\text { Av. }\end{array}$ & $\stackrel{r}{r}$. o & $\begin{array}{l}r \xi \\
1 . .\end{array}$ & - & rer.r.r. & $\varepsilon$ & $\begin{array}{l}\text { ry } \\
\text { Nv.o }\end{array}$ & $\stackrel{r}{r}$. & $\begin{array}{c}17 \\
17.9\end{array}$ & 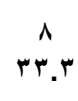 & $\begin{array}{c}r \mu \\
q 0 . \wedge\end{array}$ & $\begin{array}{l}* 1 \\
* \star \leqslant .1\end{array}$ & $Y \&$ & $\begin{array}{c}\text { Staphylococcus } \\
\text { aureus }\end{array}$ \\
\hline ir. & $r v^{3}$ & $\begin{array}{l}17 \\
1 . .\end{array}$ & - & $\begin{array}{l}1 \varepsilon \\
\wedge v .0\end{array}$ & r & $\begin{array}{l}\text { ir } \\
\text { vo }\end{array}$ & ro & ar.v & $\therefore$ & $\begin{array}{l}19 \\
1 . .\end{array}$ & - & $\begin{array}{c}1 \leqslant \\
\text { NV.O }\end{array}$ & rir. & $\begin{array}{l}1 \xi \\
\text { Av. }\end{array}$ & or & 10 & $\therefore$ & $\begin{array}{l}14 \\
1 .\end{array}$ & - & 17 & $\begin{array}{c}\text { Pseudomonas } \\
\text { aeruginosa }\end{array}$ \\
\hline 1. & - & $\begin{array}{c}1 \\
1 .\end{array}$ & - & r.r. & 19.9 & 19.8 & rr.r & צ'. & r.r. & & 18.9 & $\begin{array}{l}1 \\
1 .\end{array}$ & $\cdot$ & צ'. & r.r. & or. & s. & r.r. & 19.8 & 4 & $\begin{array}{c}\text { Escherichia } \\
\text { coli }\end{array}$ \\
\hline vo & ro & $\stackrel{\varepsilon}{1 .}$ & - & ro & ro & ro & ro & $\stackrel{r}{r}$. & $\dot{r}$ & 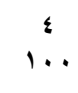 & - & r. & r. & ro & ro & ro & ro & $\stackrel{\varepsilon}{1 .}$ & - & $\varepsilon$ & Klebsiella spp. \\
\hline $\begin{array}{r}r \\
1 .\end{array}$ & - & $\begin{array}{c}r \\
1 .\end{array}$ & - & $\stackrel{r}{1 . .}$ & - & - & $\stackrel{i}{1 . .}$ & $\therefore$ & $\therefore$. & $\stackrel{r}{i .}$ & - & $\begin{array}{r}r \\
1 .\end{array}$ & - & $\therefore$ & $\therefore$. & $\begin{array}{r}r \\
1 .\end{array}$ & - & $\begin{array}{r}r \\
1 .\end{array}$ & - & r & Proteus spp. \\
\hline
\end{tabular}




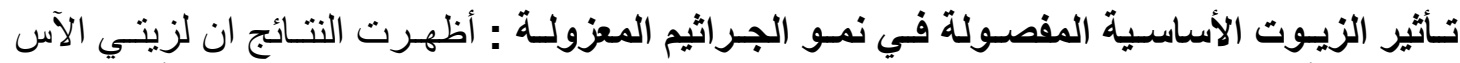

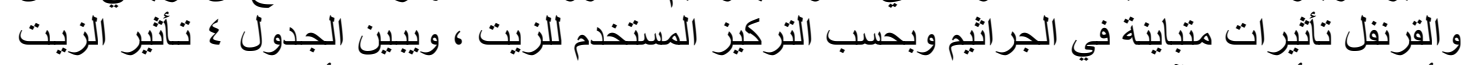

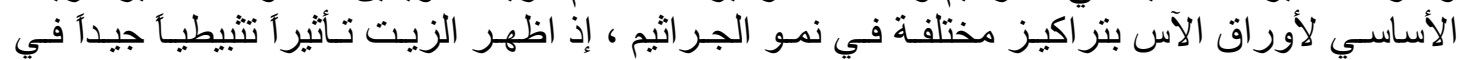

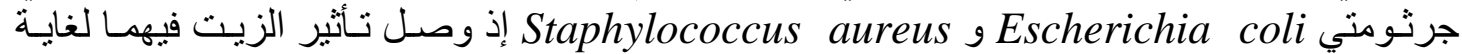
التركيز 0. . • حجم/حجم في حين وصل تأثنير الزيت في جراثيم Pseudomonas aeruginosa و و

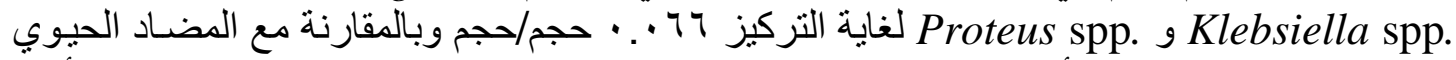

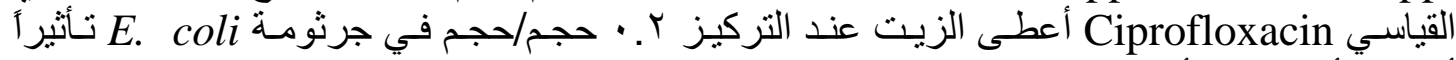

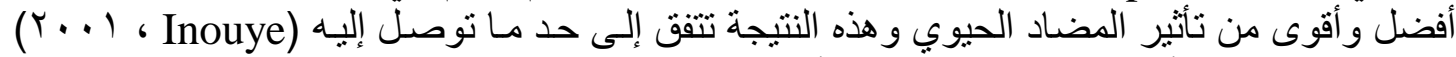

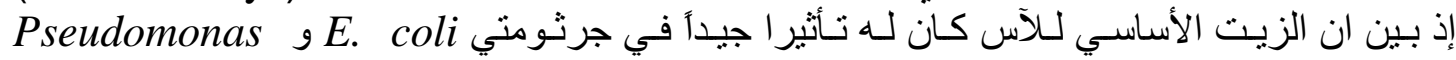
aeruginosa

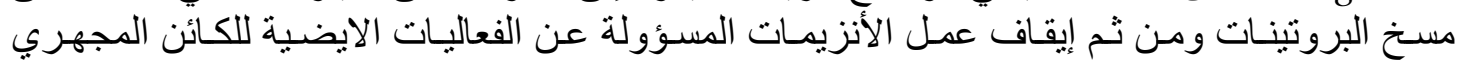

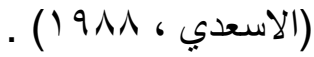

الجدول (ع) : تأثثر الزيت الأساسي لأوراق الآس في نمو الجراثيم المعزولة من الحروق (قطر دائرة

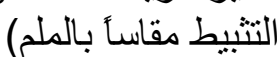

\begin{tabular}{|c|c|c|c|c|c|}
\hline $\begin{array}{c}\text { Staphylococcus } \\
\text { aureus }\end{array}$ & $\begin{array}{c}\text { Pseudomonas } \\
\text { aeruginosa }\end{array}$ & $\begin{array}{c}\text { Escherichia } \\
\text { coli }\end{array}$ & $\begin{array}{l}\text { Klebsiella } \\
\text { spp. }\end{array}$ & $\begin{array}{c}\text { Proteus } \\
\text { spp. }\end{array}$ & التركيز حجم/حجم \\
\hline . & $\cdot$ & $\cdot$ & 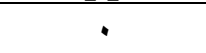 & 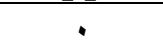 & $\because r \wedge$ \\
\hline - & . & - & - & - &.$\mu r$ \\
\hline$\cdot$ & $\cdot$ & $\cdot$ & . & $\cdot$ & $\because \varepsilon \cdot$ \\
\hline 1. & . & 1. & - & . & $\because 0$. \\
\hline IY & 1. & $1 \pi$ & 1. & 1. & $\because 77$ \\
\hline 17 & $1 \pi$ & 10 & $\pi$ & IT & $\because 1 \cdots$ \\
\hline 11 & 10 & 11 & 17 & 17 & $\cdot r \ldots$ \\
\hline 19 & 11 & IV & $r \cdot$ & r & $\begin{array}{c}\text { Ciprofloxacin } \\
\left(\diamond_{\mu g} / \text { disk }\right)\end{array}$ \\
\hline
\end{tabular}

يوضح الجدول (0) تأثثير الزيت الأساسي للقرنفل بتر اكيز مختلفة على الجر اثيم المعزولة ، إذ وإ

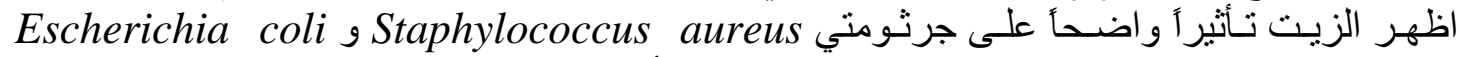

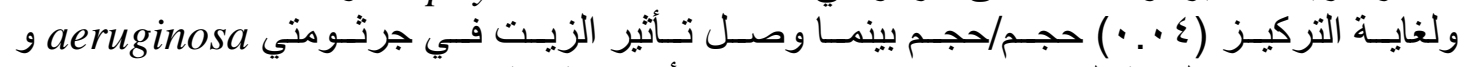

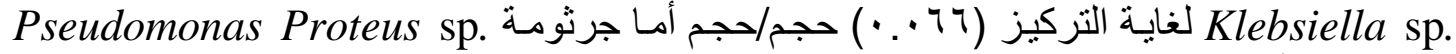

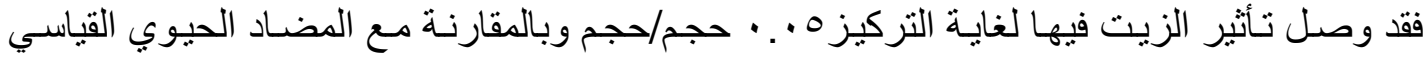

Ciprofloxacin

الجدول (0) : تأثير الزيت الأساسي للقرنفل في نمو الجراثيم المعزولة من الحروق (قطر دائرة التثبط

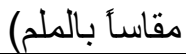

\begin{tabular}{|c|c|c|c|c|c|}
\hline $\begin{array}{c}\text { Staphylococcus } \\
\text { aureus }\end{array}$ & $\begin{array}{c}\text { Pseudomonas } \\
\text { aeruginosa }\end{array}$ & $\begin{array}{c}\text { Escherichia } \\
\text { coli }\end{array}$ & $\begin{array}{l}\text { Klebsiella } \\
\text { spp. }\end{array}$ & $\begin{array}{l}\text { Proteus } \\
\text { spp. }\end{array}$ & حجم/حجم \\
\hline$\cdot$ & $\cdot$ & $\cdot$ & 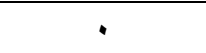 & . & $\because r \wedge$ \\
\hline • & . & . & . & . & 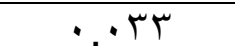 \\
\hline 1. & . & Ir & . & . & $\because \varepsilon$ \\
\hline$\pi$ & . & $1 \varepsilon$ & . & Ir & $\because 0$ \\
\hline 10 & $\pi$ & 17 & Ir & $1 \leqslant$ & $\because 77$ \\
\hline 11 & 10 & 19 & 10 & 17 & $.1 \ldots$ \\
\hline$r$. & IV & YI & 11 & 19 & 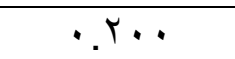 \\
\hline 19 & 11 & IV & $r \cdot$ & TY & Ciprofloxaci \\
\hline
\end{tabular}




\begin{tabular}{|l|l|l|l|l|l|}
\hline & & & & & $\mathrm{n}\left({ }^{\circ} \mu \mathrm{g} / \mathrm{disk}\right)$ \\
\hline
\end{tabular}




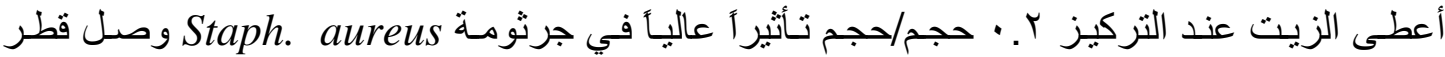

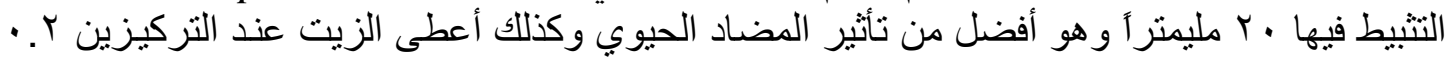

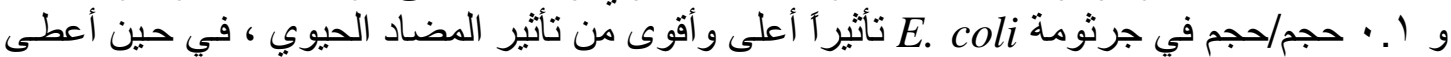

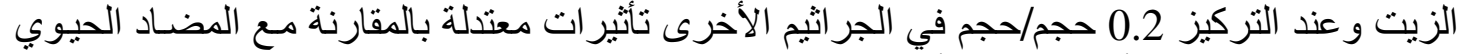

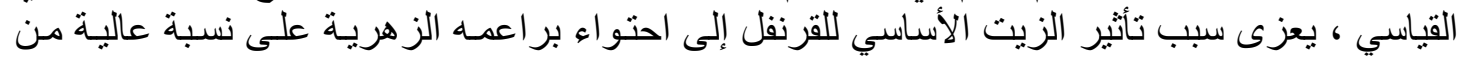

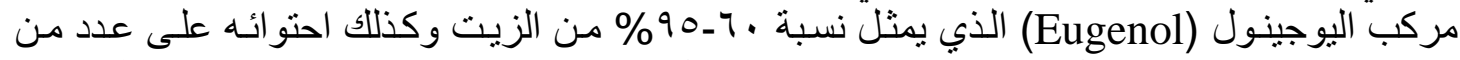

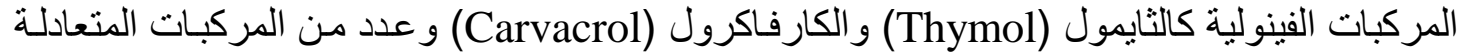

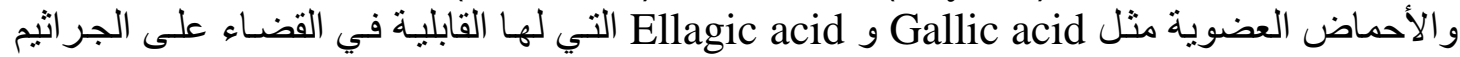

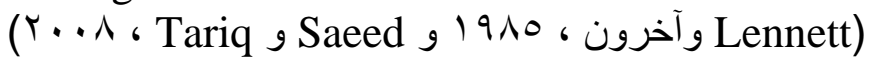

\title{
INHIBITORY EFFECT OF Myrtus communis AND Eugenia caryophyllata ESSENTIAL OILS ON GROWTH OF BACTERIA ISOLATED FROM BURNS OF UNDER FIVE YEARS CHILDREN
}

Ibraheem Talal Al-Saoaf

Nursing College / Mosul University

\begin{abstract}
Isolation and identification of bacteria that contaminated burns in children under the age of five years in both genders treated in Al-Zahrawy hospital for the period from September to November 2009 of Seventy samples collected from burns and cultured on blood agar, 52 isolates were identified and included 24 isolates $34.28 \%$ were Staphylococcus aureus, 16 isolates $22.85 \%$ were Pseudomonas aeruginosa, 6 isolates $8.57 \%$ were Escherichia coli, 4 isolates $5.71 \%$ were Klebsiella spp. and two isolates $2.85 \%$ were Proteus sp. The study also included extraction of essential oils from Myrtus communis and dried flower buds of Eugenia caryophyllata. The inhibiting effects of these oils were investigated on growth of bacteria that isolated from burns using disk diffusion method. The essential oils showed good inhibiting effects on the bacteria and the essential oil of Eugenia caryophyllata was better than Myrtus communis essential oil.

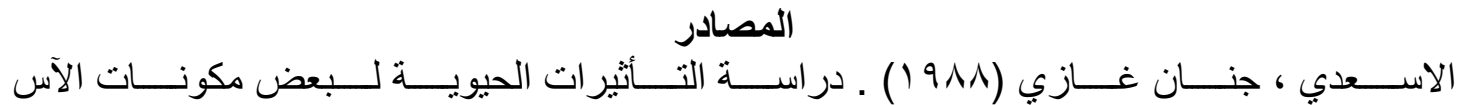

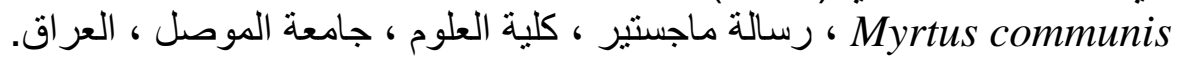

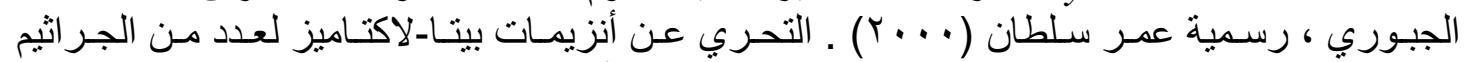

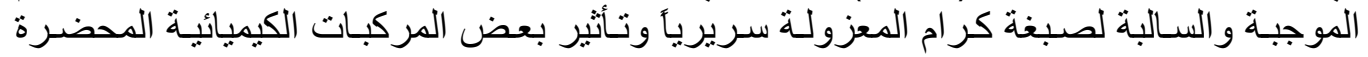

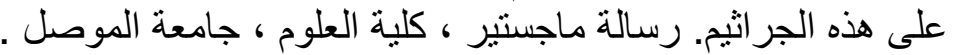

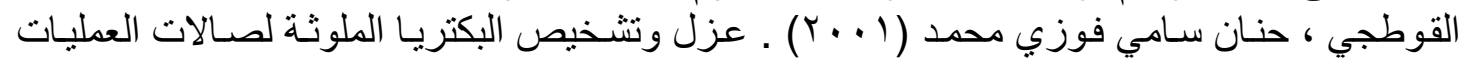

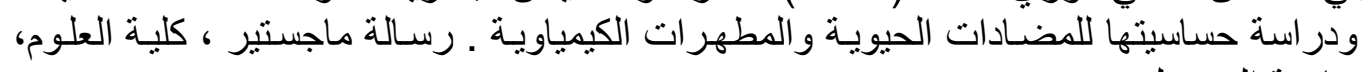

$$
\begin{aligned}
& \text { جامعة الموصل. }
\end{aligned}
$$

Alshimary, I. E. (2009). Eiffcacy of some antibacterial agents on staphylococcus aureus isolated from various burn cases. J. Med. Sci., 1(4):110-114.

Bauer, A.W.; W.A.M. Kirbay; J.S. Sherries and M. Turk (1966). Antibiotics susceptibility testing by a standardized single disk method. Am. J. Clin. Patho., 45:495-496. 
Betoni, J. E.; R. P. Mantovani; L. N. Barbosa; L. C. De-stasi and F. A. Junior (2006). Synergism between plant extract and antimicrobial drugs used on staphylococcus diseases. Mem. Inst. Oswaldo Cruz. 101(4):387-390.

Chakravarty, H. L. (1976). Plant Wealth of Iraq, A dictionary of Economic Plants, 1. Botany Directorate, Ministry of Agriculture and Agrarian Reform, Baghdad, Iraq.

Chen, T. Y.; H. F. Shang; T. L. Chen; C. P. Lin; C. F. Hui and J. Hwang (1999). Recombinant protein composed of pseudomonas exotoxin A, Outer membrane proteins I \& F asvaccine against $P$. earuginosa infection. Appl. Microbiol. Biotechnol., 52: 524-533.

Diflore, M. S. H. and V. P. Eroschenko (1989). Atlas of Normal Histology. 6thed Lea \& Febiger, Philadelphia, London, 85-87.

Donald, L. P.; M. L. Gary; S. K. Georgs and G. E. Randall (1990). Introduction To Organic Laboratory Technigues Amiroscale Approach, Sanndrs College, Publishing, pp.48-667

Doyoub, A.; F. Zeidan and S. Radidy (1995). Infection in burns experience of teaching hospital in Syria. Ann. Medit. Burns. Club., 1: 1-4.

Earss, Management team (2002). Suceptibility of Staphylococcus aureus. EARSS Newslett., 4:4-5.

Ekrami, A. and E. Kalantar (2007). Bacterial infections in burn patients at a burn hospital in Iran. J. Med. Res., 126: 541-544.

Evans, W. C. (1997). Trease and Evan's "Pharmacology". 4thed, W. B. Saunders Com., UK .

Fallon, M. T.; W. Shafer and E. Jacob (1999). Use of cefazolin microsperes to treat localized methicillin-resistant Staphylococcus aureus infection in rats. J. Sury. Res., 86(1) : 97-102.

Finegold, S. M. and E. J. Barson (1986). Baity and Scotts Diagnostic Microbiology, 7thed. Mosby Co. St. Louis, 90-95.

Inouye, S; T. Takizawa and H. Yamaguchi (2001). Antibacterial activity of essential oils and their major constituents against respiratory tract pathogens by gaseous contact, J. Antimicrobial Chemotherapym 47 : 565-573.

Kolmes, H. J. (1999). Carriers of Staphylococcus aureus as asource of nosocomial infections. epidemological and prophyloctic aspects. Ugeskr. Laeger., 161(11): 1580-1584.

Komolafe, O. O.; J. James and L. Kalongolera (2003). Makoka M. bacteriology of burns at the Queen Elizabeth central hospital, Blantyre, Malawi. Burns. 1-4.

Koneman, E.W.; S.D. Allen; W.M. Janda; P.C. Screckenberger and W.C.W. Winn (1997). Color Atlas and Textbook of Diagnostic Microbiology. 5th ed., J. B. Lippincott - Raben Publishers, Philadelphia, 37-45.

Lari, R.; H. H. Bahrami and R. Alaghehbandan (1998). Pseudomonas infection in Tohid burns center, Iran. Burns., 24: 637-641.

Lennette, E. H.; A. Balows; W. J. Hausler and H. J. Shadomy (1985). Manual of Clinical Microbiology. 4thed. American, Soc. Microbiol. Washington, USA. 
Liwimbi, O. M. and I. O. Komolafe (2007). Epidemiology and bacterial colonization of burn injuries in Blantyre. M. Med. J. 19(1): 25-27.

Miranda, G; N. Castro; C. Leanos; A. Valenzuela; U. Graza-Ramos; T. Rojas; F. Solorzan; Chichu and J. Silva (2004). A clonal and horizontal dissemination of Klebsiella pneumonias expressing SHV-5 extendedspectrum B-lactamase in Mexican pediatric hospital. J. Clin. Microbiol., 42(1) : 30-35.

Paint, D. V.; M. A. Gore; Salieshwar; Deodhar (1993). Laboratory data from the surveillance of a burns word for the detection of hospital infection. Burns., 19: 52-54.

Pichon, N.; M. J. Joseph and J. Raynaud (1993). 3-beta-D-Myricetin of Myrtus communis plant, Phytotherapic J., 26(2) : 86-90.

Prashar, A.; I. C. Locke and C. S. Evans (2006). Cytotoxicity of clove (Syzygium aromaticum) oil and its major components to human skin cells. Cells Prolif. 39 : 241-248.

Pruitt, B. A.; A. T. Manus; S. H. Kim and C. W. Goodwin (1998). Burn wound infections : current status world. J. Sur., 22(2) : 135-145.

Rahman, M. and S. Coule (2002). Antibactrial activity of hydrodistilled essential oils of Psammageton canescens. J. Biotech. 1(1) : 55-60.

Saeed, S. and P. Tariq (2008). In vitro antibacterial activity of clove against gram negative bacteria. Pak. J. Bot. 40(5):2157-2168.

Solomakos, A.; A. Govaris; P. Koidis and N. Botsoglou (2008). The antimicrobial effect of thyme essential oil, nisin and their combination against Listeria monocytogenes in minced beef during refrigerated storage. Food Microbiol., 25 : 120-127.

Thaler, F.; J. E. Rohan and P. Loirate (1998). Pseudomonas aeruginosa infection in burn patients. J. Med. Mal. Infec. 28: 167-174.

Uwaezuoke, J. C. and L. E. Aririatu (2004). A survey of antibiotic resistant Staphylococcus aureus strains from clinical sources on owerri. J. Appl. Sci. Environ. 8(1) : 67-69.

Vandepitte, J.; K. Engbreak; P. Piot and C.C. Heuck (1991). Basic Laboratory Procedure in Clinical Bacteriology. World Health Organization, Geneva

Wiener, J.; J. P. Quinn; P. A. Bradford; R. V. Gooring; C. Nathan; K. Bush and R. A. Weistien (1999). Multiply antibiotic resistance Klebsiella and Escherichia coli in nursing homes. J. Am. Med. Assoc. 281(6): 517523.

Wildemanee, C.; C. Godard; G. Vershragen; G. Claeys and C. Duyck (2004). De beenhouwer $H$. Ten years phage typing of Belgian clinical methicillin-resistant Staphylococcus aureus isolates. J. Hosp. Infect. 56: 16-21.

Wisplinghoff, H.; H. Seifert; M. Coimbra; R. P. Wenzel and M. B. Edmond (2001). Systemic inflammatory response syndrome in adult patients with nosocomial blood stream infection due to Staphylococcus aureus . J. Clin. Infec. Dis. 33: 733-736. 
Yalcin, A. W.; M. Hayran and S. Unal (1997). Economic analysis of nosocomial infections in a Turkish university hospital. J. Chem. 9(6): 411-414.

Yang, Y. C.; S. H. Lee; D. H. Choi and Y. J. Ahn (2003). Ovicidal and adulticidal effects of Eugenia caryophylata bud and leaf oil compounds on Pediculus capitis. J. Agric. Food Chem.. 51(17) : 4884-4888. 